\title{
Approche théorique de la gouvernance des organisation non lucratives
}

\section{A theoretical approach to the governance of nonprofit organizations}

\section{Bernard Enjolras}

Numéro 314, octobre 2009

URI : https://id.erudit.org/iderudit/1020965ar

DOI : https://doi.org/10.7202/1020965ar

Aller au sommaire du numéro

Éditeur(s)

Association Recma

ISSN

1626-1682 (imprimé)

2261-2599 (numérique)

Découvrir la revue

\section{Citer cet article}

Enjolras, B. (2009). Approche théorique de la gouvernance des organisation non lucratives. Revue internationale de l'économie sociale, (314), 63-83. https://doi.org/10.7202/1020965ar

\section{Résumé de l'article}

Cet article développe une approche théorique de la structure de gouvernance des organisations non lucratives. Celles-ci sont analysées comme structures de gouvernance renforçant la norme de réciprocité (généralisée ou équilibrée) et rendant possible la mutualisation des ressources. Leur structure de gouvernance présente des traits spécifiques relatifs aux finalités formelles de l’organisation, à sa forme de propriété, à ses prétendants résiduels, à ses procédures de prise de décision, à ses formes d'imputabilité, à sa division des pouvoirs, à ses procédures de contrôle, ainsi qu'aux incitations qu'elle génère. Ces traits facilitent l'action collective orientée vers l'intérêt général, l'intérêt mutuel ou l'action tribunitienne. Les organisations non lucratives sont à même d'opérer dans des environnements complexes, mobilisant des ressources provenant d'opérations marchandes, de subventions publiques ou de la réciprocité (bénévolat, dons), tout en poursuivant des objectifs civiques ou démocratiques. Leur structure de gouvernance leur permet de demeurer comparativement plus efficaces que d'autres formes organisationnelles, même si les organisations non lucratives peuvent connaître des échecs susceptibles de saper leur capital-confiance et leur efficacité. 


\section{APPROCHE THÉORIQUE DE LA GOUVERNANCE DES ORGANISATIONS NON LUCRATIVES}

* Institute for Social Research, Oslo. Mél. : bernard.enjolras@samfunns forskning.no.

** Une première version de cette contribution, présentée au colloque Addes du 10 mars 2009, est consultable sur www.addes.asso.fr.

\author{
par Bernard Enjolras*
}

Cet article développe une approche théorique de la structure de gouvernance des organisations non lucratives. Cellesci sont analysés comme structures de gouvernance renforçant la norme de réci procité (généralisée ou équilibrée) et rendant possible la mutualisation des ressources. Leur structure de gouvernance présente des traits spécifiques relatifs aux finalités formelles del'organisation, à $₫$ formede propriété, à sesprétendants résiduels, à ses procédures de prise de décision, à ses formes d'imputabilité, à sa division des pouvoirs, à ess procédures de contrôle, ainsi qu'aux incitations qu' elle génère. Ces traits facilitent l'action collective orientée versl'intérêt général, l'intérêt mutuel ou l'action tribunitienne Lesorganisations non lucratives sont à même d'opérer dans des environnements complexes, mobilisant des ressources provenant d'opérations marchandes, de subventions publiques ou de la réciprocité (bénévolat, dons), tout en poursuivant des objectifs civiques ou démocratiques. Leur structure de gouvernance leur permet de demeurer comparativement plus efficacesque d'autres formes organisationnelles, même si les organisations non lucratives peuvent connaîtredeséchecssusceptiblesde saper leur capital-confiance et leur efficacité**.

es principales théories économiques de l'organisation non lucrative, en termes d'échec public (Weisbrod, 1977) et d'échecs du marché (H ansmann, 1980; Ben-N er et Van H oomissen, 1994), expliquent pourquoi les organisationsnon lucratives, plutôt que les organisationslucra tives ou publiques, produisent certains biens et services. La théorie économique définit les organisations non lucratives à partir d'une de leurs caractéristiques: la contrainte de non-distribution du profit.

Cependant, comme le notent plusieurs contributeurs (Ben-N er et Gui, 2003; O rtmann et Schlesinger, 2003; Steinberg, 2006), la contrainte de non-distribution, d'une part, ne garantit pas que les organisations non lucratives soient dignes de confiance et, d'autre part, ne suffit pas à caractériser ces organisations. O rtmann et Schlesinger (2003) ont identifié trois défis que les théoriciens dela contrainte de non-distribution doivent relever afin de démontrer qu'elle constitue une condition suffisante garantissant que les organisations non lucratives sont dignes de confiance: 
la compatibilité des incitations, l'adultération des finalités et l'ubiquité de la réputation. La condition de compatibilité des incitations implique que les comportements opportunistes et frauduleux sont effectivement encadrés par la contrainte de non-distribution. La condition de nonaltération des finalités requiert que la contrainte de non-distribution soit suffisante pour éviter le déplacement des finalités et des objectifs de l'organisation. Enfin, le défi de l'ubiquité de la réputation oblige les tenants de la contrainte de non-distribution à démontrer pourquoi la réputation fonctionne pour les organisations non lucratives et pas pour les organisations lucratives.

D ifférentes contributions ont cherché à dépasser la notion de contrainte de non-distribution. Ainsi, $\mathrm{H}$ ansmann (1987) affirme le rôle du contrôle de la part des « patrons » de l'organisation sur le comportement organisationnel et Ben-N er (1986) souligne l'importance du contrôle exercé par les consommateurs comme un moyen de réduire les problèmes d'asymé trie d'information. Cependant, la théorie de l'organisation non lucrative souffre encore de l'absence d'un cadre général d'analyse permettant d'expliquer comment les organisations non lucratives sont à même defaire face aux échecs publics et à ceux du marché. Cet article a pour objet de tracer les contours d'une telle approche théorique concevant l'organisation non lucrative comme une structure de gouvernance (W illiamson, 1996) adaptée à la coordination de l'action finalisée et capable de résoudre différents types d'échec de coordination : échec public, échec du marchéet échec del'action collective.

\section{Mécanismes de coordination}

(1) Deux composantes des coûts de transaction sont habituellement distinguées : une dimension comportementale, la rationalité limitée et l'opportunisme, et une dimension transactionnelle, la présence d'incertitude et la fréquence des transactions. Lorsque les coûts de transaction sont élevés, il est plus efficace de recourir à la hiérarchie (l'organisation) qu'au marché. (2) L'action décentralisée d'une multitude d'agents peut être coordonnée par le jeu de différents mécanismes de coordination comme le marché ou la hiérarchie.
Les organisations sont des acteurs collectifs caractérisés par leur capacitéà centraliser la prise de décision (EIster, 2007, p. 427). Les organisations non lucratives partagent cette caractéristique, mais diffèrent des organisations publiques et des organisations lucratives dans la mesure où leur structure de gouvernance (finalités formelles, propriété, distribution des droits de propriété, procédures de prise de décision) est adaptée aux exigences de l'action collective et permet la mutual isation des ressources et la production d'un capital de confiance. D e ce point de vue, les organisations non lucratives ont recours à la hiérarchie en tant que moyen de coordination de leurs parties prenantes. Coase (1937) et Williamson $(1975,1985)$ ont montré qu'en présence de coûts de transaction (1) la hiérarchie est susceptible d'être un mécanisme de coordination plus efficace que le marché. Les organisations non lucratives, de même que les autres types d'organisation, opèrent au sein d'environnements complexes caractérisés par différents mécanismes de coordination (2) (Enjolras, 2000). Elles sont impliquées dans différents types de transaction, ayant recours à divers types de ressources. Par exemple, Weisbrod (1998a, 1998b) considère les organisations non lucratives comme des organisations multi-produits qui ont 
la possibilité de produire trois types de bien : un bien collectif préféré (l'output liéà la mission del'organisation), un bien privé préféréet un bien privé non préféré (source potentielle de revenus finançant l'output lié à la mission del'organisation). D ecepoint devue, les biens privés sont produits afin de subventionner le bien collectif.

Les organisations non lucratives sont donc à même de réaliser trois types de transactions (Polanyi, 1957a; Polanyi 1957b; Zelizer, 1998): des transactions commerciales, où la monnaie est utilisée comme compensation en échange de biens et de services; des transactions fondées sur un droit de créance, où monnaie, biens et services sont alloués selon un droit prédéfinissant la règle d'allocation; et des tran sactions réciprocitaires, où monnaie, biens et services sont mutuellement échangés sur la based'une normede réciprocité (Gouldner, 1960; Fehr et Gächter, 1998).

Chaque type de transactions correspond à un type de ressources pour les organisations non lucratives. Les financements publics mettent en jeu des transactions fondées sur un droit de créance, les dons et le bénévolat sont fondés sur des transactions réciprocitai res, tandisque les revenus de la vente de biens et de services impliquent des transactions commerciales. Une organisation non lucrative «pure » est par conséquent une organisation qui tire ses ressources des dons et du bénévolat, c'est-à-dire qui implique uniquement des transactions réciprocitaires. Parallèlement à cetype 0 «pur », trois autres types d'organisations non lucratives peuvent être identifiés (Enjolras, 2002) en fonction de leurs ressources dominantes. Le type « commercial » est caractérisé par la prédominance de ressources marchandes. Letype «tiers partiefinancement » est liéà la domination des fonds publics dans les ressources del'organisation. Letype «économie solidaire » combine les ressources marchandes, publiques et réciprocitaires. Ces principes de transaction peuvent aussi être conçus en termes demécanismes de coordination (tableau 1, en page suivante).

\section{La réciprocité}

Le premier mécanisme de coordination à considérer est celui de l'action collective. Les organisations non lucratives peuvent être pensées en termes de " mécanisme de mutualisation » (H orch, 1994). Elles permettent I'association de personnes ayant des intérêts partagés et rendent possible la mutualisation de ressources afin de poursuivre ces intérêts. De ce point de vue, les organisations non lucratives réalisent la coordination d'acteurs sur la base de l'intérêt mutuel. La mutualisation de ressources suppose aussi uneforme de réciprocité. Commeles dons ou letravail volontaire, ces types de transaction se conforment à une « normegénéralede réciprocité » (Gouldner, 1960; Kolm, 2006) définie comme " un échange mutuellement contingent de gratifications entre deux ou plusieurs unités». L'obligation de rendre un service ou d'être reconnaissant envers celui qui l'a procuré apparaît comme un puissant mécanisme de coordination pour les relations personnelles mettant en jeu la confiance, ainsi que pour les relations non personnelles telles que les dons et le bénévolat. 
L'existence de l'H omo reciprocans (Fehr et Gächter, 1998) a été rapportée dans plusieurs expérimentations utilisant la théorie des jeux, suggérant qu'« une importante fraction de la population a la volonté de récompenser les actessympathiques et de punir les acteshostiles». D ans les sociétés primitives, la connexion entre flux matériels et relations sociales est susceptible d'impliquer un mouvement de biens, mais unetransaction donnée, inversement, suggère une relation sociale particulière (M auss, [1923] 1991). Comme le souligne Sahlins (1972, p. 187), le domaine des transactions réciprocitaires peut être réduit en deux types: premièrement, les mouve ments entre deux parties connus comme réciprocité et, deuxièmement, les mouvements centralisés qui impliquent la collecte et la mutualisation des ressourcesau sein d'un groupe. Cependant, d'un point de vue plus général, les deux types fusionnent, dans la mesure où la mutual isation est une organisation des réciprocités. La mutualisation est une relation àl'intérieur d'un groupe, l'action collective d'un groupe, tandis que la réciprocité est une relation entre individus, l'interaction de deux parties. Pour Sahlins (1972, p. 190-193), la notion de réciprocité qualifie une classe entière d'échange, une continuité de formes. A une extrémité du continuum se trouvent l'assistance offerte librement, les relations familiales quotidiennes, l'amitié et les relations de voisinage, le «don pur » de M alinowski. La "réciprocitégénéralisée », qui renvoieaux transactions altruistes (assistance donnéeet si possible retournée), constituele point solidaire du continuum. A l'autre extrême, la « réciprocité négative », ou l'insociabilité, est caracté risée par l'essai d'obtenir quelque chose en échange de rien en toute impunité. Au point médian du continuum se situe la « réciprocité équilibrée », référant à l'échange direct où les valeurs échangées séquilibrent.

\section{Tableau 1}

Mécanismes de coordination dans les organisations non lucratives

\begin{tabular}{l|l|l|l}
$\begin{array}{l}\text { Mécanisme } \\
\text { de coordination }\end{array}$ & Marché & Action collective & Etatique-coercitif \\
\hline $\begin{array}{l}\text { Principe } \\
\text { de coordination }\end{array}$ & Prix & $\begin{array}{l}\text { Intérêt mutuel, } \\
\text { norme } \\
\text { de réciprocité }\end{array}$ & Loi, coercition \\
\hline $\begin{array}{l}\text { Mécanisme } \\
\text { d'allocation }\end{array}$ & Marché & Mutualisation & Redistribution \\
\hline $\begin{array}{l}\text { Echec } \\
\text { de coordination }\end{array}$ & $\begin{array}{l}\text { - Externalités } \\
\text { - Asymétries } \\
\text { informationnelles } \\
\text { - Biens publics }\end{array}$ & $\begin{array}{l}\text { - Passager clandestin } \\
\text { - Réciprocité négative }\end{array}$ & $\begin{array}{l}\text { Insatisfaction } \\
\text { des préférences }\end{array}$ \\
\hline
\end{tabular}




\section{Le marché}

Le deuxième mécanisme de coordination impliquant les organisations non lucratives est celui du marché. D ans le cadre de l'échange marchand, les valeurs sont échangées sur la base de prix négociés ou de prix déterminés par le jeu de l'offre et de la demande (Polanyi, 1957b). Pour les économistes, chaque acteur se comporte en fonction du signal donné par le prix. D ans lemodèle del'équilibre général entre la demande (les consommateurs) et l'offre (les producteurs), le marché est un mécanisme autorégulé.

\section{L'Etat}

Le troisième mécanisme est la coordination étatique-coercitive. Elle est associée aux transactions fondées sur un droit de créance où les valeurs échangées sont déterminées par les lois et règlementations recourant à la coercition pour leur mise en œuvre.

\section{Echecs de coordination}

Chaque mécanisme de coordination correspond à un mécanisme d'allocation qui caractérise la manière dont les ressources sont dirigées et distribuées dans l'économie. Ce mécanisme est susceptible de rencontrer trois types d'échec: échec du marché, échec public et échec de l'action collective.

\section{Echec du marché}

Dans le cas de l'échec du marché, différents phénomènes tels que le hasard moral, la sélection adverse ou l'impossibilité d'écrire des contrats contingents sont en jeu. Tous ces phénomènes résultent de l'incertitude relative soit au comportement d'une partie prenante de l'échange, soit à la qualité de l'objet échangé. H abituellement, cette incertitude est liée à l'asymétrie informationnelle entre les parties. La sélection adverse caractérise les situations où l'incertitude concerne l'objet échangé, tandis que le hasard moral concerne l'incertitude relative à l'action d'une des parties. D ans ce cas, l'objet de l'échange est constitué de l'action d'une des parties. Cette action n'est pas directement observable par l'autre partie, ce qui est source d'incertitude. Par ailleurs, dans le cas de transactions de long terme, les contrats ne peuvent pas anticiper l'ensemble des cas de figure susceptibles d'advenir. La présence d'un savoir spécifique ou la nécessité d'investissements irréversibles constituent égal ement une source d'échec du marché. En présence d'externalités, les acteurs coordonnés n'atteignent pas un résultat socialement optimal du fait des interdépendances non prises en compte par le mécanisme de coordination (les prix). Enfin, dans le cas de biens publics, la nature de l'objet, caractérisée par l'indivisibilité et la non-exclusi vité, ne permet pas aux transactions de prendre place et les agents n'ont aucune incitation à se coordonner (passager clandestin). 


\section{Echec public}

Les biens publics purs possèdent deux caractéristiques distinctives: il n'est ni possibleni désirable d'exclure ou de rationner leur usage. La nature deces biens est telle qu'il est très difficile ou très coûteux de rationner leur usage. En effet, leur usage par un consommateur additionnel ne restreint pas I'usage des autres consommateurs (le coût marginal de production est nul). Les biens publics soulèvent le problème du passager clandestin: I'usager n'est inciténi à payer pour l'usage du bien ni à révéler ses préférences, dans la mesure où il sait pouvoir en bénéficier librement. En conséquence, le marchééchoue, puisqu'il n'existe aucune incitation pour que le bien soit offert sur une base volontaire. D e nombreux biens ne sont pas des biens publics purs (possédant les caractéristiques de non-exclusion - indivisibilité- et denonrivalité dans l'usage), mais des bienstutélaires possédant l'une des deux caractéristiquesà des degrés variables, soit parcequel'exclusion est possiblemais coûteuse, soit parce qu'elle est réali sable mais non désirable.

La fourniture publique de biens publics ou tutélaires (c'est-à-direl'allocation résultant d'un mécanisme de coordination étatique-coercitif) peut s'avérer non optimale dans la mesure où les agents n'ont aucune incitation à révéler leurs préférences et qu'il serait trop coûteux voireimpossible pour la puissance publique d'obtenir cette information.

\section{Echec de l'action collective}

Si les organisations non lucratives sont conçues comme des systèmes permettant d'organiser sur une base privée l'action collective coordonnée à partir d'un principe de réciprocitégénérale ou équilibrée, elles sont sujettes au problème de l'action collective tel que formulé par O Ison (1965). L'action collective ne se produit que si le bénéfice net de la contribution de chaque individu est positif, ce qui suppose des incitations sélectives. Cependant, le problèmedel'action collective met en œurreune autredimension qui peut être approchée à partir du dilemme du prisonnier ( $\mathrm{H}$ ardin, 1992). M ême si chaque individu réalise un bénéfice net positif en contribuant à l'action collective, pour un individu donné la meilleure stratégie est de ne pas contribuer, quelle que soit l'action des autres individus. $\mathrm{Ne}$ pas contribuer (donc se comporter en passager clandestin) est la meilleure stratégie, quelle que soit la stratégie des autres.

Jusque là nous avons distingué trois types de mécanismes de coordination susceptibles d'être mobilisés par les organisations non lucratives dans le cadre de leurs activités. Chacun de ces mécanismes de coordination rencontre des limites. La principale question, maintenant, consiste à déterminer comment les organisations non lucratives sont en mesure de résoudre ces échecs de coordination. Comme le souligne W eisbrod (1977), l'action collective mise en œuvre au sein des organisations non lucratives constitue une manière de faire face aux échecs publics. Lorsque l'offre de biens publics par les autorités publiques conduit à l'insatisfaction des consommateurs (certains étant sur-satisfaits, d'autres sous-satisfaits), la production de biens publics par les organisations non lucratives est susceptible 
de satisfaire les demandes hétérogènes ou minoritaires. II reste cependant à déterminer comment les organisations non lucratives résol vent les échecs du marché (s'agissant des biens de confiance) ainsi que ceux de l'action collective. L'argument développé dans cet article est que la structure de gouvernance spécifiquequi caractérise les organisations non lucratives leur permet de résoudre les échecs du marché et ceux de l'action collective, et par conséquent de constituer une alternative à l'offre publique de biens collectifs. Afin de développer cet argument nous procédons en deux étapes. N ous allons tout d'abord voir comment la prise en compte dela normede réciprocité réduit l'importance des problèmes d'action collective. N ous démontrons ensuite comment la structure de gouvernance des organisations non lucratives favorise la réciprocité et la confiance.

\section{Action collective et norme de réciprocité}

Si le fait de ne pas contribuer à l'action collective (comportement de passager clandestin) est la meilleure stratégie quel que soit le comportement des autres contributeurs à l'action collective, le fait d'introduire la possibilité de se comporter conformément à une norme (par exemple la norme de réciprocité) conduit à une conclusion différente: elle est susceptible de réduire les problèmes d'action collective (H olländer, 1990; Koford et M iller, 1991; Kollock, 1998; Lindbeck et al., 1996; Kübler, 2001; H echter et O pp, 2001; Feld, 2002).

Lorsque l'on ne se conforme pas à un principe moral internalisé ou à une norme (Sherif, 1966; Scott, 1971; Elster, 1989, 1990, 1999), un processus de dissonance cognitive génère des sentiments de honte et de culpabilité. La dissonance cognitive apparaît lorsqu'une distance est créée entre ce que I'on devrait faire (obéir à une norme donnée) étant donné son statut social (et sa conception de soi-même) et ce que l'on fait, c'est-à-dire ce que l'on communique à propos de soi aux autres (ne pas obéir à la norme). La distance entre sa perception subjective de la façon dont on est perçu par les autres (en termes de statut social ou d'identité) et sa propre déclaration à propos de la personne que l'on est (amour-propre) crée une dissonance. Selon Festinger (1957), la dissonance, psychologiquement inconfortable, motive la personne à se rapprocher de la consonance. Les psychologues s'accordent sur l'importance de l'internalisation des règles ou normes de comportement. Les sociologues, quant à eux, soulignent le rôle dela pression sociale dans les processus de conformité normative. Lorsqu'une personne a internalisé un principe ou une norme, de même que lorsqu'un principe ou une norme sont mis en œuvre par un groupe dont la personne est membre, la perspective d'une action allant à l'encontre de la norme crée une dissonance entre ce que la personne doit faire et ce qu'elle a I'intention de faire. La dissonance créera des sentiments d'anxiété, de culpabilité et de honte. L'individu est susceptible de réduirela dissonance en choisissant une action qui est consonante avec la norme. 
Contrairement au dilemmedu prisonnier, où la stratégie dominante pour les deux joueurs est la défection (ne pas coopérer), l'introduction d'un comportement de conformitéà une norme conduit à une solution coopé rative. Q uel est l'effet del'introduction d'une norme de coopération dans le cadre des dilemmes sociaux (comme le jeu du prisonnier) lorsque l'information est incomplète, c'est-à-dire lorsque les joueurs ne savent pas quel est le type des autres joueurs et lorsque la possibilité qu'un des joueurs ne soit pas égoïste (c'est-à-dire se conforme à une norme) est considérée? II est possible de démontrer que les individus égoïstes prenant en compte le fait que certaines personnes puissent se conformer à la norme de coopération peuvent avoir intérêt à contribuer. Le dilemme caractérisant la structure du jeu de bien public repose sur le fait que les deux joueurs savent qu'ils sont égoïstes. Le fait de considérer la possibilité que les joueurs puissent se conformer à une norme (comportement normatif) et que les joueurs ignorent le type de l'autre joueur change la structure du jeu. La caractéristique la plus importante de cette analyse est que les interactions stratégiques dans un contexte social «normatif », c'est-à-dire où les individus ont la possibilité d'agir en se conformant à une norme, ne conduisent pas nécessairement aux mêmes résultats que les interactions où les individus s'attendent à ce que les autres agissent à partir de leurs intérêts. Le problème de l'action est résolu et la coopération est soutenue par la norme de réciprocité lorsque les bénéfices anticipés de la contribution à I'action collective sont suffisamment élevés, même si certains individus agissent de façon égoïste.

\section{Structure de gouvemance}

Si la norme de réciprocité est susceptible de résoudre, ou à tout le moins de réduire, les problèmes d'action collective, toute solution aux échecs de coordination (Enjolras, 2000; Bowles, 2004) implique un résultat exprimé en termes d'allocation (l'avantage reçu par chaque contributeur). L'action collective fondée sur la réciprocité est incompatible avec la structure de gouvernance lucrative, dans la mesure où les individus ne sont ni incitésà contribuer à une organisation (action collective) quand une telle contribution enrichit les propriétaires de l'organisation (profits) ni susceptibles de se conformer à la norme de réciprocité lorsque le résultat (l'allocation des ressources) d'unetelle contribution est en contradiction avec la norme de réciprocité en termes de justice distributive.

Les organisations non lucratives se caractérisent par une structure de gouvernance (finalités formelles, propriété, distribution des droits de propriété, procédures de prise de décision) qui facilite et soutient l'action collective fondée sur la réciprocité. Elles fonctionnent en effet commeun mécanisme mutualiste (H orch, 1994). La mutual isation des ressources suppose un principe de réciprocité et la structure de gouvernance de ces organisations est compatible avec cette norme. 


\section{La confiance}

La structure de gouvernance de ces organisations est susceptible de renforcer la norme de réciprocité, résolvant du même coup le problème del'action collective. Cependant, I'habileté del'organisation à favoriser les comportements réciprocitaires est liée à sa capacité à inspirer la confiance. D ans le cas des transactions impliquant la confiance (C oleman, 1990), on trouve le paradigme de la décision risquée. Celui qui investit sa confiance doit choisir entre deux possibilités: investir sa confiance ou ne pas l'investir. Son choix va dépendre de son estimation de la probabilité que la personne en qui il investit sa confiance se conforme à la norme de réciprocité. Si l'incertitude concernant le comportement probable de la personne en qui la confiance est investie excède un certain seuil, la personne n'investira pas sa confiance et la transaction échouera. Comme l'illustre le modèle du donneur de $\mathrm{H}$ ansmann, l'asymétrie d'information peut conduire à l'échec d'une transaction fondée sur la réciprocité. Si l'on considère une personne qui désire accroître la quantité ou la qualité du produit d'une organisation non lucrative, mais n'est pas en mesure d'observer l'impact de son don (en temps ou en monnaie), l'incertitude relative à la façon dont son don sera utilisé peut la conduireà ne pas donner. D e ce point de vue, le problème de l'action collective fondée sur la réciprocité possède des éléments communs à celui de l'échec du marché. D ela même manière, la capacité de l'organisation à mobiliser des financements par le biais de subventions publiques ou de contrats avec les autorités publiques est lourdement dépendante de la confiance qu'inspirel'organi sation relativement à l'usage qu'elle fait des fonds publics. En conséquence, la principale question consisteà déterminer comment la structure de gouvernance de I'organisation non lucrative est en mesure de favoriser le capital de confiance dont jouit l'organisation.

Bien que la perspective développée ici s'applique à tout type d'organisation non lucrative, il est nécessaire de distinguer les organisations de membres, qui sont plus répandues en Europe, des organisations où les membres se limitent au conseil d'administration, modèle dominant aux Etats-U nis et en $\mathrm{G}$ rande-Bretagne. Les premières présentent une structure de gouvernance démocratique où les membres élisent un conseil d'administration assumant la direction del'organisation. D ans les secondes (board managed organizations, Salamon, 1997), les membres du conseil d'administration sont soit nommés par des organisations ou autorités externes à l'organisation, soit cooptés et auto-perpétués.

Chaque forme institutionnelle, lucrative, publique ou non lucrative, peut être caractérisée par une structure de gouvernance qui lui est spécifique. Six dimensions de la structure de gouvernance peuvent être identifiées (tableau 2, en page suivante) : finalités de l'organisation, propriété, distribution desdroits de propriété, en particulier les prétendants résiduels, façon dont le pouvoir de décision est distribué, mécanismes de contrôle et d'imputabilité, incitations encastrées dans la structure de gouvernance. 


\section{Tableau 2}

Structures de gouvemance et formes organisationnelles (types idéaux)

\begin{tabular}{|c|c|c|c|}
\hline & Lucrative & Publique & Non lucrative \\
\hline $\begin{array}{l}\text { Finalités } \\
\text { organisationnelles }\end{array}$ & $\begin{array}{l}\text { - Profit } \\
\text { - Action } \\
\text { économique }\end{array}$ & $\begin{array}{l}\text { - Intérêt général- } \\
\text { bien public } \\
\text { - Action orientée } \\
\text { économiquement }\end{array}$ & $\begin{array}{l}\text { - Intérêt général } \\
\text { ou mutuel } \\
\text { - Action politique } \\
\text { - Action orientée } \\
\text { économiquement }\end{array}$ \\
\hline Propriété & Privée & Publique & Collective \\
\hline $\begin{array}{l}\text { Prétendants } \\
\text { résiduels: } \\
\text { - Contrôle résiduel } \\
\text { (iusabutend) }\end{array}$ & $\begin{array}{l}\text { - Propriétaires- } \\
\text { actionnaires }\end{array}$ & - Etat & $\begin{array}{l}\text { - M embres-conseil } \\
\text { d'administration } \\
\text { (avec droits } \\
\text { d'aliénation } \\
\text { limités) }\end{array}$ \\
\hline $\begin{array}{l}\text { - Revenu résiduel } \\
\text { (iusfinuend) }\end{array}$ & $\begin{array}{l}\text { - Propriétaires- } \\
\text { actionnaires }\end{array}$ & - Etat & $\begin{array}{l}\text { - Absence } \\
\text { de prétendants } \\
\text { résiduels } \\
\text { sur les revenus } \\
\text { (contrainte de } \\
\text { non-distribution) }\end{array}$ \\
\hline Prise de décision & $\begin{array}{l}\text { Conseil } \\
\text { d'administration- } \\
\text { assemblée générale } \\
\text { des actionnaires } \\
\text { en proportion } \\
\text { de la part de capital }\end{array}$ & $\begin{array}{l}\text { M embres du conseil } \\
\text { d'administration } \\
\text { nommés par les } \\
\text { autorités publiques }\end{array}$ & $\begin{array}{l}\text { M embres } \\
\text { du conseil } \\
\text { d'administration } \\
\text { cooptés ou élus } \\
\text { par l'assemblée } \\
\text { générale } \\
\text { des membres }\end{array}$ \\
\hline $\begin{array}{l}\text { Mécanismes } \\
\text { de contrôle } \\
\text { et d'imputabilité }\end{array}$ & Capitaliste & Démocratique & $\begin{array}{l}\text { Démocratique } \\
\text { et/ou division } \\
\text { des pouvoirs }\end{array}$ \\
\hline $\begin{array}{l}\text { Incitations } \\
\text { encastrées }\end{array}$ & Extrinsèques & $\begin{array}{l}\text { Extrinsèques } \\
\text { et intrinsèques }\end{array}$ & $\begin{array}{l}\text { Extrinsèques } \\
\text { et intrinsèques- } \\
\text { réputation }\end{array}$ \\
\hline
\end{tabular}




\section{Intérêt général versus lucrativité}

La première dimension concerne les finalités formelles de l'organisation. Le principal objectif des organisations lucratives est le profit, tandis queles organisations publiques ont pour objectif la poursuite de l'intérêt général ou l'offre de biens publics. Les organisations non lucratives, quant à elles, poursuivent la réalisation soit de l'intérêt général ou de l'intérêt mutuel, soit de fins politiques. D e plus, elles n'ont pas comme finalité première l'activitééconomique. Weber (1978, p. 64) distingue «l'action économique» et «l'action économiquement orientée», qui est «primairement orientée vers d'autres finali tés et qui prend en compte dans la poursuite de ces finalités des considérations économiques». Les activités économiques sont celles visant à produire et à consommer des biens et des services, tandis que les activités économiquement orientées visent d'autres finalités, mais impliquent la production et la consommation de biens et de services pour la réalisation de ces finalités. Une part significative des activités mises en œuvre par les organisations non lucratives appartient à cette dernière catégorie. La majeure partie des activités dans les domaines de l'éducation, de la santé, des services sociaux, de l'art et de la culture, de la philanthropie, du sport et de la religion n'ont pas la production et la consommation de biens et de services comme finalité première. La forme non lucrative et sa structure de gouvernance sont adaptées à l'organisation d'activités économiquement orientées, impliquant des objectifs axiologiques et mettant en œuvre une allocation des ressources fondée sur la réciprocité. Les final ités axiologiques et instrumentales diffèrent dans la mesure où les activités axiologiques n'ont pas pour final ité la réal isation d'un avantage ou d'un intérêt, mais la réalisation de valeurs non monétaires, tandis que le marché ne valorise que ce qui peut être quantifié et exprimé en termes monétaires.

C ependant, la vie sociale ne peut être réduite aux valeurs monétaires. Les êtres humains poursuivent la réalisation d'autres valeurs qui relèvent de la culture, de la solidarité, del'humanité, du prestige, de la beauté, de la démocratie, de l'intérêt public, etc., qui ne peuvent être exprimées en termes monétaires. D e nombreuses activités humaines sont orientées vers l'actual isation de ces valeurs. Leur réalisation suppose souvent la production et la consommation de biens et de services leur conférant une dimension économique sans qu'elles puissent être réduites à une valeur monétaire.

\section{Propriété privée, collective ou publique}

Les formes organi sationnelles sont aussi distinguées à partir de leur forme de propriété. D ans le cas de l'organisation lucrative, la propriété est de nature privée. Les organisations publiques sont par nature de propriété publique, tandis que les organisations non lucratives font l'objet d'une propriété collective ou prennent la formede «trusts» (Salamon, 1997). La littérature sur les droits de propriété classifie les formes institutionnelles selon les types d'arrangements contractuels qui définissent les droits sur les revenus résiduels (c'est-à-dire les revenus restants une fois que les rémunérations fixées par contrat ont été payées) et lesdroits de contrôle résiduel 
(le pouvoir de direction incluant celui d'aliénation de la propriété) [Eggertsson, 1990]. D ans les organisations lucratives, si l'on ignore les problèmes liés à la relation principal-agent entre actionnaires et managers qui sont le plus souvent réglés par l'octroi de stock-options aux managers, les propriétaires supportent les conséquences en termes de richesse de leurs actions. D ans les organisations publiques ou non lucratives, il n'existe pas de «propriétaires » qui les supportent directement. Cette caractéristique constitue le fondement des arguments cherchant à démontrer que les organisations lucratives sont plus efficientes que les organisations publiques ou non lucratives, dans la mesure où les organisations lucratives incorporent des incitations à la minimisation des coûts, ce que les autres formes institutionnelles ne font pas. II est important de préciser que cette ligne d'argumentation est fondée sur l'idée sel on laquelle les acteurs sont égoïstes et uniquement motivés par leur intérêt matériel (extrinsèque) et ne prend pas en compte le fait que d'autres mécanismes de gouvernance sont susceptibles de favoriser l'efficience.

\section{Droit de propriété et revenu résiduel}

Les organisations peuvent donc être classées en termes d'arrangements contractuels qui gouvernent la distribution de leurs « droits de propriété » et particulièrement de leur « revenu résiduel ». Cette classification met l'accent sur la relation entre les actions des décideurs et la mesure dans laquelle ces décideurs supportent les conséquences financières (en termes de richesse) de leurs décisions. Il est usuel de distinguer trois types dedroit de propriété (Eggertsson, 1990; Furubotn et Richter, 1998, p. 77):

- le droit d'usage physique de l'objet (iusutendi);

- ledroit au revenu provenant del'usage physique del'objet (iusfruendi).

- le pouvoir de direction incluant le pouvoir d'aliénation de l'objet (ius abutendi).

Uneorganisation non lucrative est caractérisée par la distribution des droits de propriété suivante:

- le droit d'usage physique de l'objet est attribué aux membresadministrateurs;

- il n'existe pas de droit aux revenus;

- le pouvoir de direction est délégué par l'assemblée générale au conseil d'administration ou attribué aux administrateurs, tandis que le pouvoir d'aliénation est limité.

En retour, cette distribution spécifique des droits de propriété définit en partie les contours du système de gouvernance des organisations non lucratives de membres, incorporant une structure de contrôle où l'assemblée générale contrôle et élit un conseil d'administration qui a la possibilité d'engager un directeur. $D$ ans les organisations uniquement composées d'administrateurs, les procédures de contrôle et d'imputabilité sont plus faibles, étant fondées sur l'intégrité des administrateurs et sur l'imputabilité vis-à-vis des parties prenantes (donneurs, bénéficiaires, etc.) et du grand public. 


\section{Responsabilité et motivation des dirigeants}

Les structures de gouvernance sont aussi définies à partir des modalités de responsabilité des dirigeants. D ans les organisations lucratives, les dirigeants sont responsables devant les propriétaires ou actionnaires. D ans les organisations publiques, les dirigeants ont un pouvoir déléguépar les autorités politiques et sont directement ou indirectement politiquement imputables devant les citoyens. D ans les organisations non lucratives de membres, les dirigeants sont responsables devant l'assemblée générale par lejeu de procédures démocratiques, tandis que dans les associations limitées au conseil d'administration, les administrateurs cooptés sont assujettisà un ensemble de règles. La séparation des pouvoirs entre les dirigeants (sal ariés) de l'organisation et le conseil d'administration est aussi susceptible de renforcer l'imputabilité par le jeu de procédures internes de contrôle. Le dernier élément caractérisant les structures de gouvernance est le type d'incitations que ces structures incorporent et qui motivent les dirigeants. Les organi sations lucratives offrent des incitations monétai res, instrumentales et extrinsèques à leurs dirigeants, prenant la forme de stockoptions, bonus fondés sur le profit, etc. Les dirigeants des organisations publiques ou non lucratives sont motivés par des incitations à la fois moné taires, instrumentales et extrinsèques et des incitations axiologiques et intrinsèques. D es valeurs comme celles de service public, d'intérêt géné ral, de bien commun jouent un rôle important, mais souvent sous-estimé, comme facteur de motivation pour les dirigeants des organisations publiques ou non lucratives. D e plus, les membres du conseil d'administration sont aussi confrontés à des incitations les motivant à maintenir leur réputation comme «bons citoyens » et administrateurs désintéressés.

\section{Structure de gouvemance et confiance}

Chacune des dimensions de la structure de gouvernance des organisations non lucratives doit être évaluée au regard de sa contribution à la confiance (trusworthiness) qu'inspirel'organisation. D ans cette perspective, les défis formulés par 0 rtmann et Schlesinger (2003), particulièrement la question dela compatibilitédes incitations et celle de la non-altération des objectifs, constituent une grille d'analyse utile.

\section{Compatibilité des incitations}

Selon le critère de compatibilité des incitations, il est nécessaire de démontrer pour quelles rai sons les opportunités et les incitations conduisant à différentes formes de comportements opportunistes et fraudul eux sont négligeables dans les organisations non lucratives.

Cette question est importante, car comme l'a souligné $H$ ansmann (1980) relativement à l'explication en termes d'échecs du marché, dans une situation d'asymétrie d'information, les organisations non lucratives sont considérées plus dignes de confiance que les organisations lucratives. 
D ans le modèle de $H$ ansmann, le producteur détient plus d'informations sur le produit que le consommateur et il est incité à tricher sur la qualité du produit. La contrainte de non-distribution (l'absence de prétendant résiduel) contribue à réduire les incitations à minimiser la qualité afin d'accroître le profit et assure le consommateur d'un certain niveau de qualité. Le problème est que la contrainte de non-distribution ne garantit pas queles organisations non lucratives ne maximisent pas leur profit. Les organisations non lucratives peuvent en effet maximiser leur profit pour satisfaire d'autres objectifs que la rémunération des actionnaires. $D$ ans ces conditions, pourquoi ces organisations serai ent-elles plus dignes de confiance?

Une autre ligne d'argumentation met l'accent sur le fait que les organisations non lucratives sont caractérisées par l'absence de prétendant résiduel. Comme le montrent Fama et Jensen (1983), l'absence de prétendant résiduel est susceptible de conduire à l'inefficience, al ors que la séparation entre propriété et contrôlegénère une situation de principal-agent. Lorsque les droits de propriété sont atténués, les membres ne peuvent ni sapproprier le surplus ni capitaliser la valeur de la firme en vendant leurs droits aux revenus futurs (Frech III, 1976; Frech III et G insburg, 1981). Les droits de propriété atténués conduiraient alors à des comportements différents de ceux induits par les droits de propriété privés, dans la mesure où ils réduisent le prix des avantages managériaux (avantages non salariaux dont profitent les dirigeants et/ou les membres). Selon cet argument, ces avantages non pécuniaires incluent d'autres éléments que la qualité et la quantité de l'output et l'organisation non lucrative présentera des coûts de production plus élevés que ceux de l'organisation lucrative.

\section{Altération des objectifs}

Une organisation peut être considérée comme une structure de gouvernance (valeurs, finalités formelles, normes et règles définissant le fonctionnement et la direction de l'organisation) et une structure sociale (comportements et interactions entre participants). Ces deux structures sont interdépendantes, mais pas identiques.

Le principal problème de ce point de vue est de déterminer dans quelle mesure la structure de gouvernance est suffisante pour garantir que l'organisation ne se détourne pas de ses final ités formelles, ce que Panebianco (1982) appelle le « dilemme entre le modèle rationnel et le modèle du sys tème naturel ». Selon le modèle rationnel, les organisations sont l'instrument de la réalisation d'objectifs spécifiques. Le système naturel considère les organisations comme des structures qui répondent et s'ajustent à une multitude de demandes émanant de nombreuses parties prenantes. Selon cette dernière conception, les final ités officielles de l'organisation ne sont qu'une façade derrière laquelle se cache la finalité réelle, qui est la survie de l'organisation et la perpétuation de ses dirigeants. II faut cependant souligner que les finalités formelles ou officielles ne peuvent pas être réduites à une façade, dans la mesure où elles font partie d'une idéologie qui est 
nécessaire au maintien de l'identité de l'organisation aux yeux de ses supporters (membres, donateurs, financeurs, etc.). C ela n'empêche pas que, dans le mêmetemps, I'organisation soit susceptible de développer des tendances à l'autopréservation et à la diversification sous les pressions de son environnement.

En résumé, les inefficiences, la réduction de la qual ité, le mauvais usage des ressources, le déplacement des finalités organisationnelles sont susceptibles de caractériser les activités économiques des organisations non lucratives, tandis que la contrainte denon-distribution non seulement negarantit pas la dignité de confiance de ces organisations, mais constitue aussi la source de leurs problèmes d'efficience et de gouvernance. Cependant, comme nous allons le voir, la structure de gouvernance des organisations non lucratives repose sur des mécanismes régulateurs et des incitations qui contrecarrent, en partie, ces effets pervers. Trois principaux mécanismes régulateurs (démocratie, équilibre des pouvoirs et contrôle) et deux types d'incitations (intrinsèques et fondées sur la réputation) peuvent être distingués.

\section{Démocratie}

D ans les organisations de membres le conseil d'administration est élu par l'assemblée générale, agit par délégation et est responsable vis-à-vis des membres. Au sein d'un tel système de démocratie représentative, le principal mécanisme garantissant queles administrateurs représentent les inté rêts et les préférences des membres est la crainte que ces derniers n'utilisent de façon active les élections pour démettre de leurs postes les administrateurs. D ans la mesure où ce type d'organisation est contrôlépar les membres qui représentent directement ou indirectement les intérêts des bénéficiaires ou clients de l'organisation, le contrôle par les membres plutôt que le contrôle par les propriétai res constitue une source de dignité de confiance plus crédible que la contrainte de non-distribution. Le mécanisme de contrôle repose cependant sur la participation active des membres, tandis que l'absence de participation active est susceptible de générer une gouvernance oligarchique caractérisée par le manque de renouvellement des dirigeants, le contrôle de la part d'une minorité des ressources de l'organisation, la professionnali sation des dirigeants, ainsi qu'un niveau faible de participation à la gouvernance de l'organisation.

\section{Equilibre des pouvoirs}

La structure de gouvernance des organisations non lucratives repose sur le principede séparation des pouvoirs. D ans les organisations de membres, l'assemblée généraleélit les administrateurs, qui sont responsables del'orientation stratégique de l'organisation et qui contrôlent le directeur (sal arié). $D$ ans ce type d'organisation, le conseil d'administration est responsable devant les membres et les administrateurs ont intérêt à être réélus. Lemême principesapplique aux organisations d'administrateurs, à la différence que les administrateurs ne sont pas responsables devant les membres, mais devant le grand public. La séparation des pouvoirs entre administrateurs 
et dirigeants salariés est importanteet renforcel'imputabilitédes premiers, car le conseil d'administration n'est pas un prétendant résiduel aux revenus de l'organisation et ne bénéficie pas d'émoluments managériaux, contrairement au dirigeant salarié. Cependant, lorsqu'uneforme de collusion entre le conseil d'administration et le dirigeant salarié se produit, le système de répartition des pouvoirs échoue en ce qu'il ne parvient pas à garantir l'imputabilité et l'usage efficient des ressources. II est par ailleurs important de souligner que le principe de séparation des pouvoirs suppose la mise en œuvre de procédures de contrôle.

\section{Contrôle}

La structure de gouvernance des organisations non lucratives inclut également le principe de contrôle actif des opérations de la part du conseil d'administration. Si ce contrôle par les administrateurs est efficace, les problèmes d'efficience sont susceptibles d'être réduits (même si les problèmes liés à la relation principal-agent entre le conseil d'administration et le dirigeant ne sont pas complètement éliminés du fait de l'asymétrie d'information qui caractérise cette relation). Le contrôle et la direction active de la part du conseil d'administration sont cependant coûteux en temps et en ressources, ce qui peut conduire à l'échec de gouvernance: tendanceà l'oligarchie, collusion entre administrateurs et dirigeant salarié et manque de contrôle effectif. D eux ensembles d'incitations sont toutefois susceptibles de limiter l'occurrence des échecs de gouvernance et contribuent à ce que les parties prenantes de l'organisation se conforment aux objectifs et aux missions de l'organisation.

\section{Incitations intrinsèques}

II est possible de distinguer deux types de motivations individuelles (Frey, 1997), intrinsèque et extrinsèque. Les motivations extrinsèques sont imposées à l'individu de l'extérieur et prennent la forme de monnaieet de sanctions, tandis que les motivations intrinsèques constituent des raisons d'agir qui proviennent de la personne, par exemple le plaisir ou la satisfaction personnelle. Les motivations extrinsèques peuvent prendre le pas sur les motivations intrinsèques dans la mesure où elles affectent négativement I'estime de soi et l'auto-détermination. Les motivations extrinsèques recouvrent souvent les motivations instrumentales, alors que les motivations intrinsèques sont souvent de nature axiologique (orientées vers des valeurs). L'engagement personnel dans les organi sations non lucratives, que ce soit comme bénévole, membre, administrateur ou employé, est plus souvent motivé par des motivations intrinsèques et axiologiques que par des motivations extrinsèques et instrumentales (M usick et Wilson, 2008).

\section{Réputation}

Un autre type d'incitation susceptible de prévenir l'échec de gouvernance est le désir de la part desadministrateurs de maintenir leur réputation ainsi que celle de l'organisation. D ans les organisations d'administrateurs, les 
administrateurs sont souvent choisis sur la base de leur expérience, leur notabilitéet leur intégrité. Leur capital social et leur réputation constituent un actif qu'ilsne souhaitent pas gaspiller. Dans les organisations demembres, s'ajoute à ces incitations le fait que les administrateurs sont imputables devant l'assemblée des membres et sont soumis à réélection. Ils sont par conséquent confrontés à une incitation additionnelle qui limite leurs comportements opportunistes et frauduleux, le désir de conserver leur position.

En résumé, il apparaît que la faiblesse des incitations extrinsèques et instrumentales qui caractérise la structure de gouvernance non lucrative et qui est souvent invoquée comme une source d'inefficience de ces organisations est compensée par différents mécanismes contraignants (démocratie, équilibre des pouvoirs, contrôle) et par des incitations (extrinsèques: la réputation) qui, considérés ensemble, lorsqu'ils sont mis en œuvre de façon efficace, garantissent la gouvernance efficiente et le capital confiance de ces organisations. Comme c'est le cas pour tout système de gouvernance, la structure de gouvernance des organisations non lucratives n'est pas exempte des risques d'échec de gouvernance ni des problèmes résiduels de gouvernance (Ben-N er et Van H oomissen, 1994). L'insuffisance de participation démocratique, les tendances oligarchiques, le manque de contrôles adé quats et de respect des standards éthiques et réglementaires, la collusion, l'éviction des incitations intrinsèques par les incitations extrinsèques, les comportements opportunistes sont susceptibles d'affecter le fonctionnement de la structure de gouvernance et, en dernière échéance, de miner la confiance dans les organisations non lucratives ainsi que leur efficience.

\section{Conclusions}

En se concentrant sur la contrainte de non-distribution, un aspect important mais partiel des organisations non lucratives, les principales approches théoriques des organisations non lucratives ont négligé une dimension cruciale de ces organisations. La conceptualisation proposéeici, mettant l'accent sur la structure de gouvernance et sur le rôle de la coordination réciprocitaire, offre un correctif à ces théories tout en les synthé tisant dans un cadre analytique unifié qui introduit la possibilité d'une pluralité de raisons d'agir (instrumentales, normatives, axiologiques), des mécanismes de coordination et des échecs de coordination.

L'accent porté sur le rôle central de la structure de gouvernance et les pratiques de gouvernance qui lui sont associées présente aussi des implications pratiques. La principale est de mettre en évidence les interdépendances qui existent entre la capacitédel'organi sation à générer des ressources et sa gouvernance. Un bon fonctionnement de la gouvernance accroît dans le même temps le capital de confiance de l'organisation, sa capacitéà faire face aux échecs de coordination, ainsi que sa capacité à générer des ressources marchandes, publiques et réciprocitaires. M algré les tensions 
qui caractérisent la gouvernance des organisations non lucratives (Cornforth, 2003), les pratiques de gouvernance incorporées dans la structure de gouvernance des organisations non lucratives est la meilleure garantie del'efficience et de la dignitéde confiance de ces organisations. Les «scandales » répétés au sein du secteur non lucratif nous rappellent que la gouvernance de ces organisations est susceptible d'échouer et que le public peut retirer sa confiance. Une conscience accrue, particulièrement du fait des administrateurs, de la centralité de la gouvernance des organisations non lucratives comme principal déterminant de la confiance qu'elles inspirent et de leur efficience peut être développée du fait d'une meilleure compréhension théorique des spécificités de la gouvernance de ces organisations ainsi que des liens fonctionnels entre échec de coordination et gouvernance interne. 


\section{Bibliographie}

Akerlof G. A., 1970, «T hemarket for lemons: quality and uncertainty and the market mechanism », Q uarterly Journal of economics, 3, p. $488-500$.

Ben-Ner A., 1986, «N onprofit organizations: why do they exist in market economies? », in S. Rose-Akerman (ed.), The economics of nonprofit institutions, 0 xford, 0 xford University Press.

Ben-Ner A., Gui B. (eds.), 1993, Thenonprofit sector in the mixed economy, Ann Arbor, The University of M ichigan Press.

Ben-Ner A., Gui B., 2003, "The theory of nonprofit organizations revisited $»$, in Anheier $\mathrm{H}$., Ben-N er A. (eds.), Thestudy of thenonprofit enterprise, N ew York, K luwer Academic.

Ben-Ner A., Van Hoomissen T., 1993, «N onprofit organizations in the mixed economy », in Ben-N er A., Gui B. (eds.), The nonprofit sector in the mixed economy, Ann Arbor, The University of M ichigan Press.

Ben-Ner A., Van Hoomissen T., 1994, "The governance of nonprofit organizations: law and public policy $»$ N onprofit M anagement and Leadership, 4:4, p. 393-414.

Bowles S., 2004, M icroeconomics: behavior, ins titutions and evolution, Princeton, Russell Sage Foundation.

Chillemi O., Gui B., 1991, «Uninformed customers and nonprofit organization », Economic Letter, 35, p. 5-8.

Coase R. H., (1937) 1952, "Thenature of the firm », in Stigler G. J., Boulding K. E. (eds.), Readings in price theory, H omewood, Irwin.

Coleman J. S., 1990, Foundations of social theory, C ambridge M A, The Belknap Press of $\mathrm{H}$ arvard U niversity Press.

Conforth C. (ed.), 2003, The governance of public and non-profit organizations, London, Routledge.

Cornes R., Sandler T., 1986, The theory of externalities, public goods and club goods,
Cambridge, Cambridge University Press.

D awson I., D unn A., 2006, «G overnance codes of practice in the not-for-profit sector », Corporate Governance, 14, p. 33-42.

Easley D., O'H ara M., 1983, «T heeconomic role of thenonprofit firm », Bell Economic Journal, 14, p. 531-538.

Easley D., O'H ara M., 1988, "C ontract and asymmetric information in the theory of the firm », Journal of economic behaviour and organisation, 9, p. 229-246.

Eggertsson T., 1990, Economic behavior and institutions, Cambridge, CambridgeUniversity Press.

Elster J., 1989, «Social norms and economic theory $»$, Journal of economic perspective, 3:4, p. 99-117.

Elster J., 1990, « N orms of revenge », Ethics, 100:4, p. 862-885.

Elster J., 1999, Alchemies of the mind, Cambridge, Cambridge University Press.

Elster J., 2007, Explaining social behavior, Cambridge, Cambridge University Press.

Enjolras B., 2000, «Coordination failure, property rights and nonprofit organizations », Annals of public and cooperativeeconomics, $71: 3$, p. 347-374.

Enjolras B., 2002, «The commercialization of voluntary sport organizations in N orway », N onprofit and Voluntary Sector Q uarterly, 31:3, p. 352-376.

Fama E. F., Jensen M. C., 1983, «Agency problems and residual claims», Journal of law and economics, 21:6, p. 327-349.

Fehr E., Gächter S., 1998, «Reciprocity and economics: the economic implications of $\mathrm{H}$ omo reciprocans», European Economic Review, 42, p. 845-859.

Feld S. L., 2002, « 0 n the emergence of social norms, review essay », Contemporary Sociology, 31:6, p. 638-640.

Festinger L., 1957, A theory of cognitive dissonance, Stanford, Stanford University Press.

Frech III H. E., 1976, «The property rights 
theory of the firm, empirical resultsfrom a natural experiment », Journal of political economy, $84: 1$, p. 143-52.

Frech III H . E., Ginsburg P. B., 1981, «Property rights and competition in health insurance: multiple objectives for nonprofit firms», Research in law and economics, 3, p. 155-72.

Frey B. S., 1997, N ot just for money: an economic theory of personal motivation, Brookfield, VT, Edward Elgar.

Furubotn E. G., Richter R., 1998, Insitutions and economic theory, the contribution of the new institutional economics, Ann Arbor, The University of M ichigan Press.

Gouldner A. W., 1960, «T he norm of reciprocity: a preliminary statement $»$, American Sociological Review, 25:2, p. 161-178.

Gui B., 1993, "Theeconomic rationale for the third sector, nonprofit and other noncapitalist organizations», in Ben-N er A., Gui B. (eds.), The nonprofit sector in the mixed economy, Ann Arbor, The University of M ichigan Press.

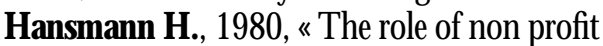
enterprise», YaleL aw Journal, 89:2, p. 835-898.

H ansmann H., 1987, «Economic theories of nonprofit organizations», in W. W. Powel (ed.), The non profit sector a research handbook, $\mathrm{N}$ ew $H$ aven, Yale University Press.

H ansmann H ., 1996, The ownership of enterprise, Cambridge M A, The Belknap Press of $\mathrm{H}$ arvard University Press.

H ardin R., 1992, Collective action, Baltimore, The Johns H opkins U niversity Press.

Hechter M., O pp K.-D . (eds.), 2001, Social norms, N ew York, Russell Sage Foundation.

H irchman A. O., 1970, Exit, voice and loyalty, C ambridge M A, H arvard University Press.

H olländer H ., 1990, "A social exchange approach to voluntary cooperation », American Economic Review, 80:5, p. 1157-1167.

H orch H. D., 1994, «O n the socio-economics of voluntary associations", Voluntas, 5-2, p. 219-230.

Knight J., 1995, «M odels, interpretations and theories: constructing explanations of institutional emergence and change ", in Knight J., Sened. I. (eds.), Explaining social institutions, Ann Arbor, TheU niversity of M ichigan Press. Koford K. J., Miller J. B. (eds.), 1991, Social normsand economic insitution, Ann Arbor, The University of M ichigan Press.

Kollock P., 1998, «Social dilemmas: theanatomy of cooperation $»$, Annual Review of sociology, 24, p. 183-214.

Kolm S. C., 2006, « Reciprocity: its scope, rationales, and consequences», in Kolm S. C., M ercier Ythier J. (eds.), H andbook of the economics of giving, altruism and reciprocity, vol. 1, Amsterdam, Elsevier.

Kübler D., 2001, « 0 n the regulation of social norms ", Journal of law, economics and organization, 17:2, p. 449-476.

Lewis D. K., 1969, Convention, Cambridge M A, H arvard University Press.

Lindbeck A., Nyberg S., Weibull J. W., 1996, «Social norms, the welfare state and voting ", seminar papers, Institute for International Economic Studies, Stockholm University.

M auss M., (1923) 1991, «Essais sur le don, formes et raison de l'échange dans les sociétés archaïques", in Sociologie et Anthrophologie, Paris, PUF.

Musick M. A., Wilson J., 2008, Volunters, a social profile, Bloomington, Indiana University Press.

National Council for Voluntary O rganizations (NCVO), 2005, Good governance: a code for the voluntary and community sector, London, N CVO. O Ison M., 1965, The logic of collective action, Cambridge M A, $H$ arvard University Press.

O rtmann A., Schlesinger M., 2003, «Trust, repute, and the role of thenonprofit enterprise», in Anheier H., Ben-N er A. (eds.), The study of the nonprofit enterprise, N ew York, Kluwer Academic.

Panebianco A., 1982, Political parties: organization and power, $C$ ambridge, Cambridge University Press. 
Polanyi K., 1957a, The great transformation, Beacon Hill, Beacon Press.

Polanyi K., 1957b, Trade and market in the early empires, N ew York, The Free Press.

Sahlins M., 1972, Stone age economics, London, Tavistock Publications.

Salamon L. M., 1987, «Partners in public service: the scope and theory of government non profit relations», in Powell W. W. (ed.), Thenonprofit sector : a research handbook, $\mathrm{N}$ ew $H$ aven, Yale University Press.

Salamon L. M., 1997, T heinternational guide to non profit law, N ew York, John W iley.

Schotter A., 1981, Theeconomic theory of social insitutions, $\mathrm{C}$ ambridge, $\mathrm{C}$ ambridge University Press.

Scott J. F., 1971, Internalization of norm, Engle wood Cliffs, PrenticeH all.

Sherif M., 1966, Thepsychology of social norms, N ew York, $\mathrm{H}$ arper Torchbooks.

Steinberg R ., 2006, «Economic theories of nonprofit organizations», in Powell W. W., Steinberg R. (eds.), Thenonprofit sector a research handbook, Second Edition, N ew H aven, Yale University Press.

Ullmann-M argalit E., 1977, The emergence of norms, 0 xford, Clarendon Press.

Von Borgstede $C_{\text {., 2002, }}$, The impact of normsin social dilemmas», thesis, department of psychology, Gothenburg University. Weber M., 1978, Economy and society, Berkeley, University of California Press.

Weisbrod B. A., 1977, The voluntary nonprofit sector, Lexington, M A, DC H eath.

Weisbrod B. A., 1988, Thenonprofit economy, C ambridge, $\mathrm{H}$ arvard University Press.

Weisbrod B. A. (ed.), 1998a, To profit or not to profit, the commercial transformation of the nonprofit sector, Cambridge, Cambridge University Press.

Weisbrod B. A., 1998b, «M odeling the nonprofit organization as a multiproduct firm: a framework for choice», in Weisbrod B. A. (ed.), To profit or not to profit, the commercial trans formation of the nonprofit sector, C ambridge, Cambridge University Press.

Williamson O. E., 1975, M arketsand hierarchies, N ew York, The Free Press.

Williamson O. E., 1985, The economic institutions of capitalism, N ew York, The FreePress. Williamson O. E., 1996, The mechanism of governance, 0 xford, 0 xford U niversity Press.

Zelizer V., 1998, «H ow do we know whether a monetary transaction is a gift, an entitlement or compensation? » in Ben-N er A., Putterman L. (eds.), Economics, valuesand organizations, Cambridge, Cambridge U niversity Press. 